\title{
Contact-free measurement of heart rate, respiration rate, and body movements during sleep
}

\author{
MARK BRINK \\ ETH Zurich, Zurich, Switzerland \\ CHRISTOPHER H. MÜLLER \\ Ergonomie \& Technologie GmbH, Zurich, Switzerland \\ and \\ CHRISTOPH SCHIERZ \\ ETH Zurich, Zurich, Switzerland
}

\begin{abstract}
We describe a noncontact method for the ambulant measurement of basic sleep physiology parameters in humans, particularly for field studies involving sleep research and sleep disturbances. This method traces the body movements, respiration, and heart action of a person at rest or asleep on a bed, using four high-resolution force sensors installed under the bedposts. The recoil movement of the body at each heartbeat, known as the cardioballistic effect, as well as the lifting and lowering of the thorax, while breathing, causes very small shifts of the center of gravity of the bed and the subject. These shifts are reflected in the altering force distributions across the four sensors. Cardiac and respiratory parameters and the subject's movement activity can be calculated from the sensor signals. Neither electrodes nor other kinds of transducers are in direct contact with the subject, which is the main advantage of this technique over conventional methods. Laboratory experiments were carried out to estimate validity and practicability. The method has been found to be adequate, especially for automated and unattended sleep-data collection over long periods of time.
\end{abstract}

In psychophysiology and sleep research, sleep quality and sleep disturbances are investigated using either rather complex measurement methods, such as polysomnography (PSG), or quite simple measurement methods, such as wrist actigraphy. Most of the methods used for field research fall in the latter category, whereas polysomnographic recordings are usually confined to the laboratory. Since the activity of the heart provides a range of psychophysiologically relevant variables, an efficient means for ambulant monitoring in the field would thus be a simple-tohandle recording system that provides not only actigraphy data, but also respiration and, in particular, heart activity parameters. Such a system would preferably also obtain

Parts of the development of seismosomnography (SSG) have been funded by the Schweizer Bundesamt für Umwelt and the Schweizer Bundesamt für Gesundheit. We acknowledge Hanspeter Honegger and Marcel Brunschwiler for their innovative work on the SSG prototype, as well as Ari Stucki and Michael Rüegg, who made many SSG recordings for validation purposes. We also thank the Hochschule für Technik Rapperswil for assistance with electronic circuit design. We thank HansRudolf Roth, Marino Menozzi, and Thommy Läubli for their statistical advice. We are also grateful to all our volunteering subjects: We appreciate their cooperation in the pilot and validation studies. Correspondence concerning this article should be addressed to M. Brink, Center for Organizational and Occupational Sciences (ZOA), LEO B7.2, ETH Zürich, CH-8092 Zürich, Switzerland (e-mail: brink@ethz.ch). the desired data in a nonintrusive way, that is, without electrodes or transducers attached to the body.

In the past decades, a range of methods for "contactfree" measuring of sleep-quality data-especially for body movements - have been developed, including photographic and video monitoring, motility beds (Crisp, Stonehill, \& Eversden, 1970; Muzet, Becht, Jacquot, \& Koenig, 1972), actigraphy watches, and movement transducers attached to the bed. But only in the more recent past have attempts been made to develop ambulant monitoring methods that do not use ECG electrodes to record parameters of heart activity. The static charge sensitive bed, introduced by Alihanka and collaborators (Alihanka \& Vaahtoranta, 1979; Alihanka, Vaahtoranta, \& Saarikivi, 1981), and later, the polyvinylidene fluoride (PVDF) mattress (Siivola, 1989), seemed to be the most successful of the noninvasive or, more precisely, noncontact techniques so far. In 1997, we started to develop our own method to unobtrusively record basic life functions during sleep. The measurement principle, technical implementation, and validation of the method will be outlined in this article.

\section{Using the Ballistocardiogram \\ to Trace the Heartbeat}

To construct a contact-free recording device for heart parameters, the ballistocardiogram (BCG) is of particu- 
lar interest. A BCG is a recording of the oscillatory body motion that occurs with every heartbeat and is therefore attributed to forces produced by the heart action. In contrast with an ECG, a BCG can be recorded by purely mechanical means. It was described as early as 1877 by J. W. Gordon, who used a horizontal wooden frame suspended from ropes and a sphygmograph (a mechanical recorder of vibrations) to trace the body displacement caused by the beating heart. The term ballistocardiogram was introduced in 1939 by Starr and colleagues (Starr, Rawson, Schroeder, \& Joseph, 1939). The genuine ballistocardiographs were constructed to assess parameters of cardiovascular dynamics such as cardiac stroke volume, but in medicine, these have long since been replaced by other methods. Today, the possibility of using the cardioballistic effect to nonintrusively trace heart activity during sleep has again stimulated interest in the $\mathrm{BCG}$ and methods for deriving it.

The cardioballistic effect occurs with each contraction of the left ventricle of the heart, whereby the blood output into the aortic arch and its subsequent acceleration in the cardiovascular system forces the body to recoil. The magnitude of this recoil movement is proportional to the volume of blood ejected from the heart per beat. A BCG records exactly this aspect of heart activity, usually when the body is positioned on a freely movable support pad. Not only the heart action, but also the lifting and lowering of the thorax and abdomen, while breathing, causes the body to displace and change its center of gravity periodically. It is possible to measure these changes in different ways. In contrast with the static charge sensitive bed, with which the amount of static charge in a mat is measured, our method records low-frequency mechanical vibrations that emanate from the sleeper and propagate through the mattress and the bed. These vibrations are picked up by force sensors under the bedposts. Making use of the Greek word seismos, for vibration, we will refer to this method as seismosomnography (SSG). In addition to heart and respiration parameters, SSG can deliver actigraphy data of a subject in bed in a more detailed fashion than wrist actimeters normally do. With our current system, we are able to record a subject's heartbeat, respiration activity, body movements, and approximate body position. In the following sections, however, we will mainly focus on the recording and analysis of heart activity and body movements.

\section{INSTRUMENTATION}

\section{Hardware}

Whereas the early ballistocardiographs were very bulky apparatuses that only operated reliably under specific laboratory conditions, an easy-to-use and field-capable ballistocardiographic recording system must function independently of a variety of factors: It must work on any type and size of bed, with different mattresses, and also with a large range of people, with varying physical characteristics. Influenced by the early attempts of Gordon and Henderson (Gordon, 1877; Henderson, 1905), who used scales or other kinds of mechanical transducers to record the cardioballistic effect, we constructed high-resolution force sensors that are installed under the four posts of a bed and carry the weight of the entire bed, as if a scale were under each post. This principle has been patented in Switzerland (Schierz, Müller, Krueger, \& Honegger, 2003). The sensors are connected to an interface that stores their signals and/or displays them on a computer screen.

Sensors. The four sensors used for SSG recordings define a 2-D coordinate system, which makes it possible to monitor the position of the center of gravity of the entire bed. The position of the center of gravity is derived from the forces applied to each sensor. Each sensor is composed of a top and bottom aluminum plate, with a reflex light barrier in between. As the force on the sensor increases, the two plates are pinched together slightly, and their clearance decreases. The reflex light barrier senses the distance between the two plates and translates it into a voltage signal that is preamplified and passed through a low-pass filter with a cutoff frequency of $4.8 \mathrm{~Hz}$, to reduce ripple and noise. In order to differentiate between the weak heart and respiration signals and the coarse body-movement signals, the preamplified electrical signal is divided into an AC and a DC component, respectively. The $\mathrm{AC}$ component is the outcome of a high-pass filter with a cutoff frequency of $0.3 \mathrm{~Hz}$. It can be amplified with a potentiometer up to a factor of 1,000, and reaches a peak-to-peak voltage of about $5 \mathrm{~V}$. The DC component results from passing the preamplified signal through a second low-pass filter, with a cutoff frequency of $7.2 \mathrm{~Hz}$. The AC component contains the very small deflections of the sensor plates around their mean distance, which, in return, is reflected by the DC component. Whereas the latter reflects the linear force on the sensor at any time, the $\mathrm{AC}$ component is particularly sensitive to acceleration and therefore able to pick up the relatively weak cardioballistic impulses and the respiration movements. Both $\mathrm{AC}$ and DC components are converted to 16-bit digital values (we use an ADS8320 A/D converter from Burr-Brown Corp.), the AC component being sampled at $100 \mathrm{~Hz}$ and the DC component at $4 \mathrm{~Hz}$. The sampled values of the four sensors are stored on disk and can be further processed by digital signal processing (DSP) algorithms. In the current prototype, amplification, filtering, and $\mathrm{A} / \mathrm{D}$ conversion take place early in the signal chain on a small circuit board mounted on the sensors themselves. A schematic drawing of a sensor is shown in Figure 1.

The sensors are constructed in a rugged fashion and can bear weights up to $100 \mathrm{~kg}$ each. The aluminum plates are made of the alloy AlMg4,5Mn0,7 (AW-5083) and are $8 \mathrm{~mm}$ thick. The sensor dimensions are $24 \times 150 \times$ $60 \mathrm{~mm}$. In order to keep the bedposts in place on top of the sensors, and to keep them from slipping off, a series of small indentations are milled on the top plates of the sensors. Small, round-headed metal pins driven into the underside of the bedposts fit in the indentations so the bed does not shift or slip off the sensors. Additional fastening methods and adapters have been constructed for beds with metal, plastic, or composite-material posts or with metal rails instead of posts. The indentations created to fixate 


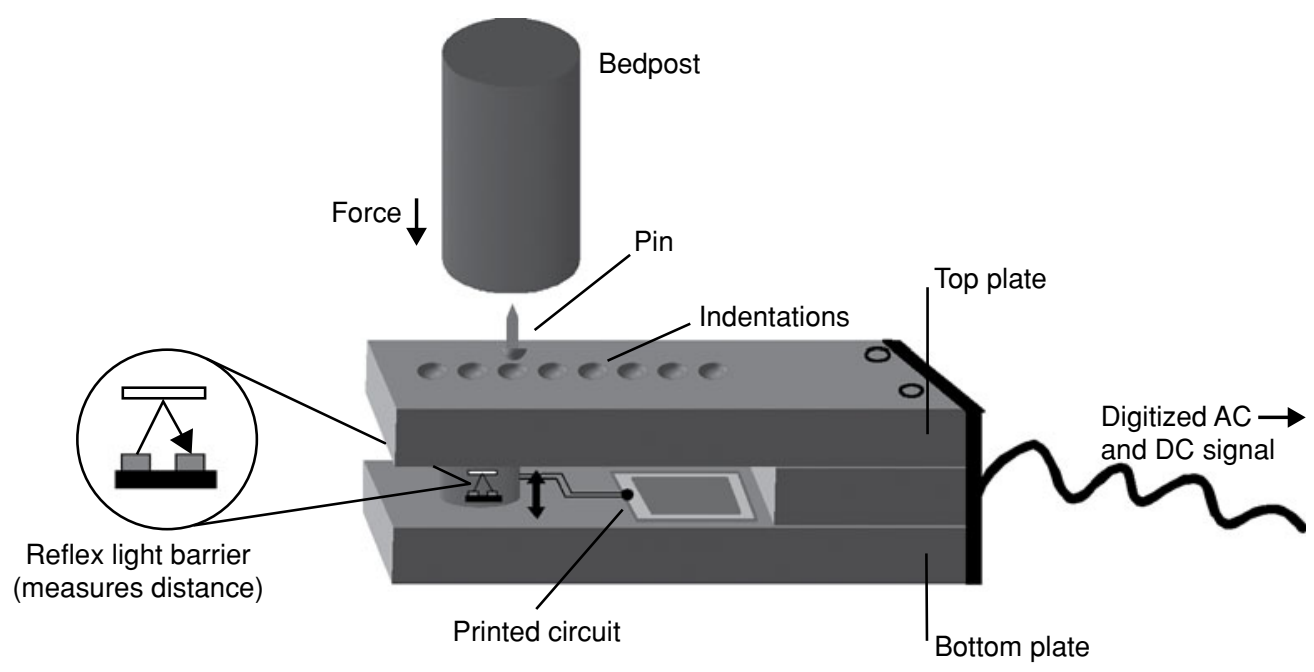

Figure 1. Schematic drawing of an SSG sensor.

the bedposts also allow for adjustment of the leverage applied on the sensor and thus its sensitivity. Independent of the type of lying position or the weight of the sleeper, the sensors always yield an AC signal from which the cardioballistic effect, in the frequency range between about 0.4 and $2.0 \mathrm{~Hz}$, and the respiratory activity, with frequencies up to $0.4 \mathrm{~Hz}$, can be derived.

Interface. The sensors connect to a small case that houses a microcontroller and a low-power subcompact board computer (Type GENE-4310, AAEON Technology Inc., Taiwan) with a 2.5-in. hard disk drive to receive and store the incoming signals (Figure 2). The interface was created without cooling fans, so it does not emit any audible noise, which is a prerequisite in sleep research. A specific service application was developed for SSG recordings that receives the digitized sensor signals via the RS232 interface from the microcontroller. The system can be programmed either to start and end recording at predefined points in time or to do so automatically as soon as the bed is weighted down by a person. The storage capacity suffices for several months of continuous recording, and the built-in Ethernet interface enables data transfer to or from a network or computer.

Approximate costs of production. The components needed to construct the system are-except for the aluminum plates that build up the sensors - available from computer and electronics suppliers. The light barrier, cables, power supply, electronic parts, and integrated circuits (A/D converters, controllers, etc.) for the main circuitry cost approximately US \$200; subcompact board computers with hard disk and RAM cost less than US \$300. The case and aluminum plates were tailored to our specifications and cost, for a complete system with four sensors, about US \$100.

\section{Signal Processing}

The four-channel raw AC signal (Figure 4, panel A) is composed of the respiratory and heart activity, oscilla- tions of the bed, high-frequency signal noise, and occasional excursions of high amplitude, which are generated by movements of the sleeper. Because of the frequencydependent amplification characteristics of the AC chain on the sensors (frequencies $>0.3 \mathrm{~Hz}$ are boosted), the respiratory amplitudes are much smaller and less apparent in the raw signal than the heart peaks. However, because a comparatively large mass is displaced while breathing, the respiratory pattern is much less vulnerable to interferences than the heart pattern. During body movements, the raw AC signal becomes too perturbed (Figure 6, panel A), and the heart action and respiration movement can no longer be detected.

The emergence of a cardioballistic signal involves many complex mechanical interactions and-depending on the bed, the lying position of the sleeper, and other factors - heart and respiration patterns may appear in different signal configurations, which cannot be known in advance. In order to obtain a signal that reflects the heart

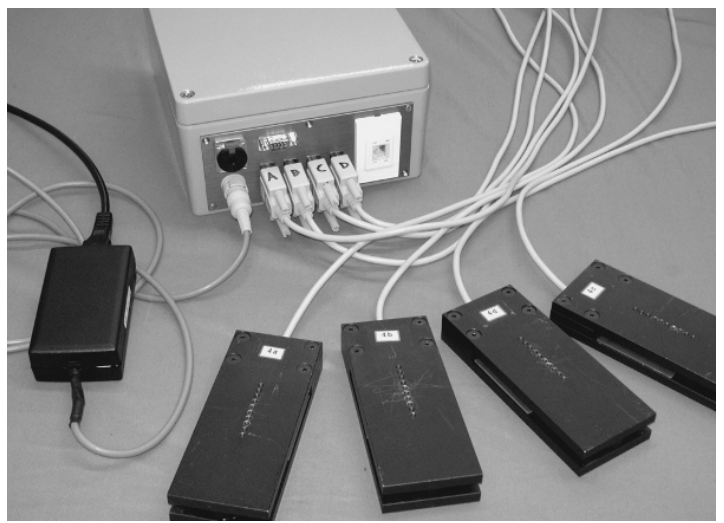

Figure 2. SSG prototype consisting of power supply, interface with front panel connectors for power supply, sensors, and Ethernet. Four sensors in the front. 
or respiration activity with the best possible clarity, we established the following procedures.

Calculation of axis coordinates. Starting from the original four-channel $\mathrm{AC}$ signal $\left(\mathrm{AC}_{\mathrm{ABCD}}\right)$, several onechannel subsignals are calculated. Of major importance are the signals reflecting the center of gravity of the bed, named $x$, for the transverse axis coordinate, and $y$, for the longitudinal axis coordinate, respectively. $x$ and $y$ represent a nonmetric approximation of the center of gravity of the bed, whose four corners (bedposts) define the 2-D coordinate system depicted in Figure 3. The values for $x$ and $y$ are calculated as follows: $x=\left(h_{\mathrm{B}}+h_{\mathrm{D}}\right)-\left(h_{\mathrm{A}}+h_{\mathrm{C}}\right)$; $y=\left(h_{\mathrm{A}}+h_{\mathrm{B}}\right)-\left(h_{\mathrm{C}}+h_{\mathrm{D}}\right)$, where $h$ denotes the $\mathrm{AC}$ or $\mathrm{DC}$ signal amplitude from the sensor at a particular corner, indexed A, B, C, or D. Both $x$ and $y$ signals can be further processed independently of each other, and both can contain aspects of the heart and respiration activity of interest (Figure 4, panel B).

Filtering. To extrude the frequency bands containing the heart and respiration patterns, the $x$ and $y$ signals are digitally filtered (for an introduction to DSP filter functions, see Smith, 1997; or Proakis \& Manolakis, 1996) according to the following steps:

(1) A linear phase low-pass filter based on the Blackman window function, with a cutoff frequency between about $2-4 \mathrm{~Hz}$, is applied to the signal.

(2) To a copy of the signal, a linear phase low-pass filter based on the same function, but with a cutoff frequency of about $0.5 \mathrm{~Hz}$, is applied.

(3) The pattern of the heart activity is calculated by subtracting the signal received in step (2) from the signal received in step (1), which results in a signal that only contains frequencies between about 0.5 and $2-4 \mathrm{~Hz}$. This signal will be referred to as Heart or Heart $_{y}$, respectively (Figure 4, panel C).

(4) The respiration pattern-referred to as $\operatorname{Resp}_{x}$ or $\operatorname{Resp}_{y}$-is already contained in the signal received in step (2) and contains frequencies up to $0.5 \mathrm{~Hz}$ (Figure 4, panel D).

If neither of the signals, Heart $x$ or Heart $y$, accurately represents the heart activity (cardioballistic movement visible), other filtered signals can be considered for the

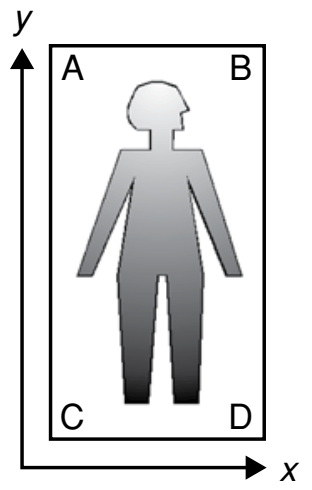

Figure 3. Layout of sensors $A, B, C$, and $D$ and orientation of $x$ - and $y$-axes. subsequent analysis-for example, the sum of the four sampled channel values or one of the two diagonal axes (AD and BC) signals.

Signal analysis. After Heart ${ }_{x}$, Heart $_{y}$, Resp $_{x}$, and Resp $y$ (or other pattern signals) are calculated, one has to decide which signal to use for further analysis. This is preferably the signal containing the most prominent heart or respiration pattern. As long as the subject is lying lengthwisewhich, in a normal bed, will almost always be the casethe heart action emerges as the so-called Head-Foot BCG, where each heartbeat produces a visible peak in the force distribution signal of the longitudinal axis of the bed. The respiratory movement-from the lifting and lowering of the thorax - also emerges in the longitudinal direction of the bed. Therefore, in most instances, heart and respiration patterns are clearly observable in the Heart $y$ and Resp signal.

Heart peaks and respiration movements are detected by an algorithm that explores the local minima (or maxima) in the signal within a moving window. The moving window is an array of an odd number of sample values continuously copied from a discrete signal. As its name implies, the window "moves" along the signal, sample by sample. For sample values in the center of the window - which are, at the same time, minimal within the window - a peak is assigned, and its position (sample number in the signal) is stored. The optimal width of the window will depend on the sample rate and the range of frequencies to be detected. It must be smaller than twice the number of samples that define the shortest allowed interpeak interval. For example, for the heart rate, with a sampling frequency of $100 \mathrm{~Hz}$ and a maximum detectable rate of $120 \mathrm{bpm}$ ( $=2 \mathrm{~Hz}=50$ sample points), the optimal window would be 99 sample points wide. To extract interpeak intervals, the number of discrete sample points between two subsequent minima in the signal are counted. On the basis of the interpeak intervals, the respiration and heart rates, as well as their variability, can be derived. In order to avoid erroneous extraction of intervals, which hampers the accuracy of heart rate measurements, the peaks must be pronounced and clearly distinguishable from random noise with large amplitudes. Even slight body movements affect the signal in such a way that no more distinct peaks can be assigned. The algorithm detects these kinds of artifacts and assigns "missing" intervals for the affected episodes.

Because the raw signal is sampled at $100 \mathrm{~Hz}$, the only technical limitation to the maximum detectable heart rate may be a result of inertia of the sleeper's body and the bed, but also the bed's higher frequency oscillations, which hamper the distinction between true heart peaks and noise components in the signal. Most of our measurements have been made using signal analysis parameters that were specified for a heart rate range of 31 to $120 \mathrm{bpm}$, corresponding to cutoff frequencies of 0.52 and $2 \mathrm{~Hz}$ and window sizes of around 100 and 400 samples, respectively. Depending on the range of heart and respiration rates that are expected in a particular measurement context, the filter and window settings can be adjusted. However, there are principal physical limitations, which 


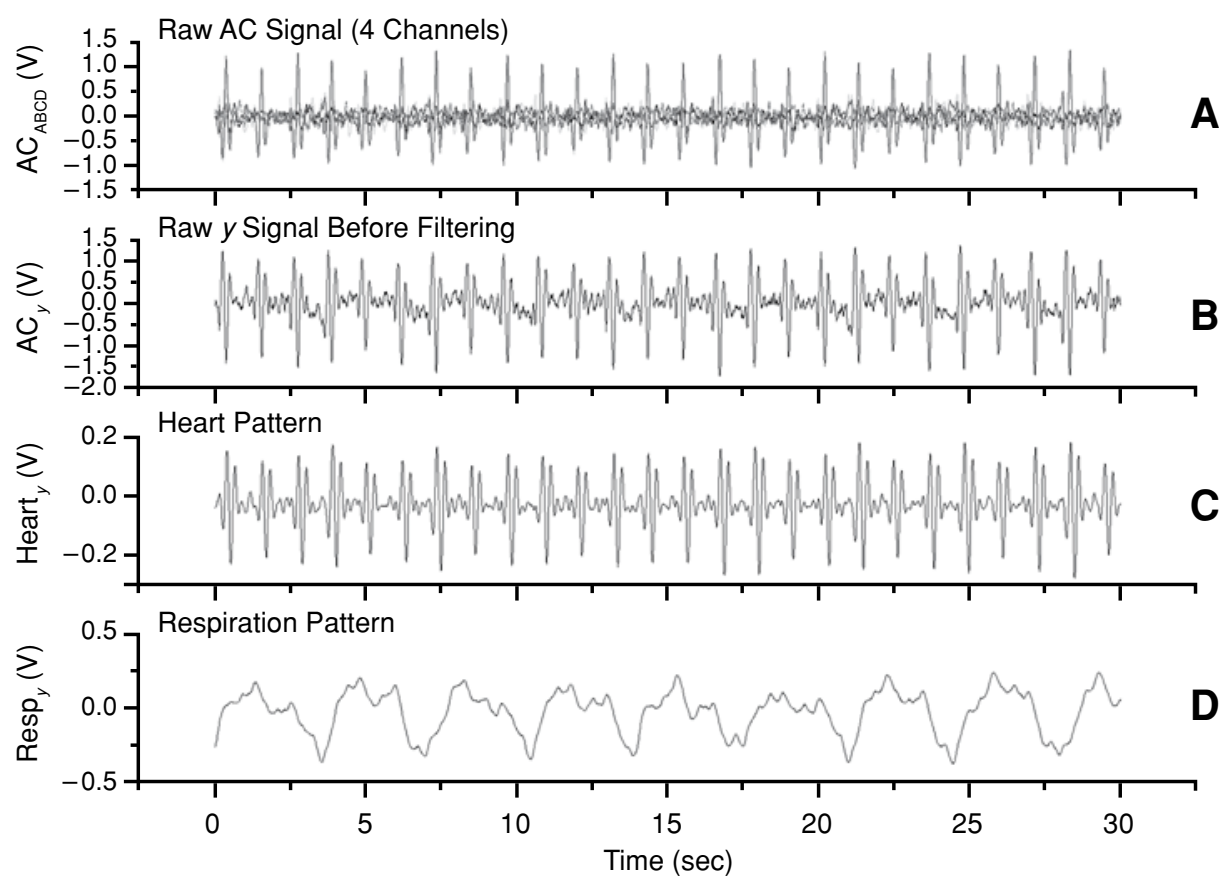

Figure 4. Example of a 30-sec SSG recording at different processing steps. (A) Raw AC signal as recorded by four sensors, A, B, C, and D. (B) Raw $y$ signal before filtering. (C and D) Heart and respiration patterns on the $y$-axis. The $y$-axis measures are in volts $(V)$.

make us believe that detecting rates higher than $120 \mathrm{bpm}$ using the cardioballistic effect is not possible — especially when higher rates are coupled with reduced blood output per beat.

\section{VALIDATION OF HEART AND RESPIRATION RATE MEASUREMENTS}

Whereas the measurement of respiration activity-a sinusoidal oscillation between head-end and foot-end force of the bed - proved easy to accomplish with any person, on any bed, reliable detection of comparatively weak heartbeats seemed to be more difficult, and extensive validation tests were required to determine the accuracy of SSG heart rate measurements. We speculated that, for example, too large and heavy a mattress might absorb an essential part of the weak kinetic energy from the heartbeat, and the cardioballistic pattern in the signal would disappear or be blurred by noise. Furthermore, because beds themselves tend to oscillate at their particular eigenfrequency, they also produce local minima or maxima in the signals, which could, under certain circumstances, be mistaken for heartbeats. Therefore, besides the subjects themselves, factors such as type and construction of the bed, stiffness and density of the mattress, and so on were also considered as possibly influencing the signal quality. To elucidate the impact of these factors on the measurement accuracy, a special device was constructed and systematic validation experiments with human subjects were carried out in the laboratory.

\section{Simulation of Cardioballistic Movements}

To systematically quantify the signal-degrading characteristics of different beds and mattresses, one must be able to produce steady and controllable cardioballistic movements on the beds. Because this cannot be achieved reliably with real subjects, we constructed a quasi-anthropometric dummy with an "artificial heart." Using this dummy, we were able to produce a synthetic cardioballistic movement with a controllable frequency and amplitude on the surfaces of mattresses of different test beds. To mimic the heart of the dummy, we employed a permanent magnet shaker (Model GWV46, Gearing \& Watson Electronics Ltd., U.K.), which was originally designed for vibration excitation on large structures (e.g., in resonance and fatigue testing of components). The shaker was driven by an $\mathrm{AC}$ amplifier at frequencies around $1 \mathrm{~Hz}$. It moved a 33-kg weight on a small cart mounted on a board, to simulate the displacement of the body by fractions of a millimeter. With a wave editor (GoldWave 4.26, GoldWave Inc., Canada) and a common PC sound card, we created several very low frequency waveforms to feed the amplifier and to control the excitation behavior of the shaker. Different waveforms were tested until a displacement movement of the dummy similar to the one caused by a natural heartbeat in a real person was realized. After an ostensibly suitable behavior of the dummy was found, it was employed to test different combinations of mattresses, frames, and beds. The agreement between the frequency at which the dummy was driven and the frequency that was measured by SSG was almost perfect (around $\pm 0.2 \mathrm{bpm}$ ) 
for all kinds of beds, frames, and mattresses. This result was promising, suggesting that the principle of measuring impulses that propagate through the mattress and the bed could also be used to derive the cardioballistic effect from humans with good accuracy.

\section{VALIDATION EXPERIMENT 1}

The purpose of the first validation experiment with human subjects was to detect systematic but undesired influences of subjects, beds, frames, and mattresses and their interactions on the validity of SSG measurements. Four subjects, three types of single beds, three types of frames, and two types of mattresses constituted a complete $4 \times 3 \times 3 \times 2$ factorial experiment (Table 1 ). The 4 subjects were chosen from 11 candidates to take part in the experiment based on their gender and heart rate at rest (measured with ECG), so that 1 male and 1 female subject with an average heart rate clearly below $60 \mathrm{bpm}$ and 1 male and 1 female subject with a heart rate clearly above $60 \mathrm{bpm}$ took part in the experiment. One of the mattresses used was made of rather stiff foam rubber, whereas the other was a soft innerspring mattress. The beds and frames were of the same size, but the beds were made of different materials: wood, metal, and plastics. All of the components to build the bed could be freely combined.

In each of the 72 different conditions, the subjects were asked to lie in a relaxed position on their backs. A 5-min SSG signal and concurrent ECG were recorded using an ambulatory ECG recorder (Model Varioport, Becker Meditec GmbH, Germany).

\section{Results}

Across all conditions, the mean heart rate measured by ECG was $57.94 \mathrm{bpm}(S D=12.60)$ and measured by $\mathrm{SSG}$, it was $57.91 \mathrm{bpm}(S D=12.57)$. To assess the amount of over- and underestimation of the heart rate by $\mathrm{SSG}$, in each of the 72 experimental conditions, the heart rate measurement value obtained by ECG was subtracted from the value obtained by SSG. This difference in measurement will be referred to as Diff ${ }_{\mathrm{SSG}-\mathrm{ECG}}$. A value of 0 indicates perfect agreement, a negative value indicates underestimation, and a positive value indicates overestimation of the heart rate, whereas the absolute difference (|Diff DSG-ECG $\mid$ ) provides a direct measure of accuracy. The mean of Diff ${ }_{\mathrm{SSG}-\mathrm{ECG}}$ was $-0.035 \mathrm{bpm}(S D=0.89$; range $=-2.67$ to 2.16$)$. The difference $\left(\right.$ Diff $\left._{\mathrm{SSG}-\mathrm{ECG}}\right)$

Table 1

Design of Validation Experiment 1

\begin{tabular}{cccc}
\hline Subject & Type of Bed & Type of Frame & Type of Mattress \\
\hline A & $\begin{array}{c}\text { old style } \\
\text { (wooden posts) } \\
\text { composite } \\
\text { B }\end{array}$ & $\begin{array}{c}\text { slatted frame } \\
\text { (soft slats) } \\
\text { slatted frame } \\
\text { (medium slats) }\end{array}$ & innerspring \\
C & $\begin{array}{c}\text { foam rubber } \\
\text { (no bedposts) }\end{array}$ & $\begin{array}{c}\text { slatted frame } \\
\text { (hard slats) }\end{array}$ & \\
D & & & \\
\hline
\end{tabular}

was not related to the mean $\left(\right.$ Mean $\left._{\mathrm{SSG}, \mathrm{ECG}}\right)(r=-.04$; $p=.72)$, but the absolute difference ( $\mid$ Diff $\left._{\mathrm{SSG}-\mathrm{ECG}} \mid\right)$ was $(r=.32 ; p=.006)$, suggesting a slightly heteroscedastic relationship. An ANOVA was calculated on $\mid$ Diff $_{\mathrm{SSG}-\mathrm{ECG}} \mid$ as dependent variable using the procedure UNIANOVA of SPSS 12.01 (SPSS Inc.). Although the beds, frames, and mattresses used in the experiment could be considered as randomly chosen representatives of their kind, they were treated as fixed factors. Subjects were treated as a random factor in the model, and the model was specified to include all main effects and all two-way interactions. Regardless of extreme values of difference, all cases were a priori included in the analysis. On the .05 alpha level, no significant main effects and no significant two-way interactions were found. The largest $F$ value was found for the factor subject $[F(3,1.17)=4.57, p=.29]$. For all effects, the probability value was greater than .19 . We could therefore not find any evidence for a difference in accuracy of measurements caused by the subjects or the characteristics of beds.

Limits of agreement. To verify whether the two methods, ECG and SSG, could be used interchangeably to a given standard of accuracy, the limits of agreement between the measurement of the heart rate by ECG and SSG were calculated - as suggested by Bland and Altman (1986) - by plotting the difference between the two measurement methods against the mean of the two methods and constructing a confidence interval for the difference (Figure 5). By subtracting measurements from each other, we removed a lot of variation between the subjects, and we could therefore expect Diff ${ }_{\mathrm{SSG}-\mathrm{ECG}}$ to be normally distributed. This assumption could be confirmed (KolmogorovSmirnov $Z=.68 ; p=.75$ ) and we would thus expect most of the differences - $95.45 \%$, to be exact - to lie between \pm 2 standard deviations from the mean difference. For the limits of agreement, defined as the range of differences within \pm 2 standard deviations from the mean, the values were $-1.82 \mathrm{bpm}(-0.035-2 \times 0.89)$ and $1.75 \mathrm{bpm}$ $(-0.035+2 \times 0.89)$, respectively.

The limits of agreement are, however, only estimates of the values that apply to the population. Therefore, the 95\% confidence intervals for the lower and upper limits of agreement $\left(\mathrm{CI}_{\text {lower }}\right.$ and $\left.\mathrm{CI}_{\text {upper }}\right)$ were calculated according to Bland and Altman (1986) by adding/subtracting the $t$-distribution value (two-tailed) for $n-1$ degrees of freedom $\times$ the standard error of the lower and upper limits of agreement, which gives

$$
\mathrm{CI}_{\text {lower }}=\overline{\operatorname{Diff}_{\mathrm{SSG}-\mathrm{ECG}}}-2 s \pm t \sqrt{\frac{3 s^{2}}{N}}
$$

and

$$
\mathrm{CI}_{\text {upper }}=\overline{\text { Diff }}{ }_{\mathrm{SSG}-\mathrm{ECG}}+2 s \pm t \sqrt{\frac{3 s^{2}}{N}},
$$

where $s$ is the standard deviation of Diff ${ }_{\mathrm{SSG}-\mathrm{ECG}}, t$ is the $t$ value for the $95 \%$ confidence interval (two-tailed) and $71 d f$, and $N$ is the sample size. 


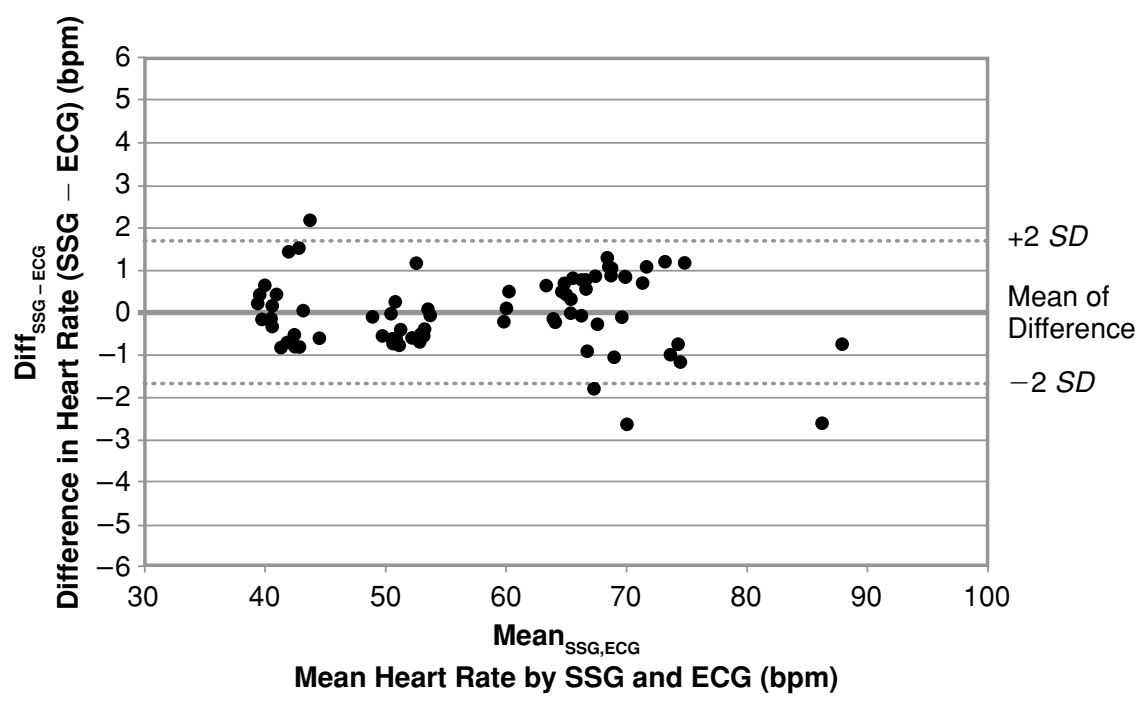

Figure 5. Difference of heart rate measurements between SSG and ECG (Diff SSG-ECG $_{\text {) }}$ from Experiment 1, plotted against their mean $\left(\right.$ Mean $\left._{\mathrm{SSG}, \mathrm{ECG}}\right)$, including $95 \%$ limits of agreement (dotted lines).

This results in:

$$
\begin{aligned}
\mathrm{CI}_{\text {lower }} & =-0.035-1.79 \pm 1.99 * 0.18 \\
& =[-2.18 \ldots-1.46]
\end{aligned}
$$

and

$$
\begin{aligned}
\mathrm{CI}_{\text {upper }} & =-0.035+1.79 \pm 1.99 * 0.18 \\
& =[1.39 \ldots 2.11] .
\end{aligned}
$$

The calculated values for the $95 \%$ confidence boundaries of the limits of agreement were -2.18 to $-1.46 \mathrm{bpm}$ and 1.39 to $2.11 \mathrm{bpm}$, respectively. Provided the experimental conditions were representative, this would be fairly accurate, at least for nonclinical purposes.

\section{VALIDATION EXPERIMENT 2}

As Validation Experiment 1 demonstrated, different beds, frames, and mattresses did not exert a notable influence on the accuracy of heart rate measurements. However, because only 4 subjects participated in Experiment 1 , we conducted a second validation experiment with a larger sample, but using only one particular bed, frame, and mattress. Also, since all measurements in Experiment 1 were made with the subjects lying on their backs, the influence of different lying positions on the accuracy of measurements still needed to be investigated and was now addressed.

Twenty-four paid subjects (12 females and 12 males) were recruited for Validation Experiment 2, in which the factors subject and lying position were examined. The mean age was 33 years $(S D=8.94$; range $=23-59)$, the mean height was $173 \mathrm{~cm}(S D=8.02$; range $=160-188)$, and the mean weight was $69 \mathrm{~kg}(S D=12.34$; range = 47-104). The subjects were asked to lie $5 \mathrm{~min}$ at a time in either a supine, sideways, or prone position on the test bed.
As in Experiment 1, the ECG was concurrently recorded and the difference between the SSG and ECG heart rate measurements was used as an indicator of accuracy.

\section{Results}

Across all 72 conditions, the mean heart rate measured by ECG was $65.38 \mathrm{bpm}(S D=8.29)$ and measured by $\mathrm{SSG}$, it was $65.7 \mathrm{bpm}(S D=8.14)$. The mean of differ-

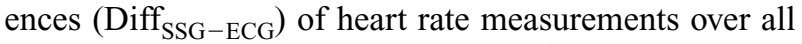
seventy-two 5-min sessions was $0.33 \mathrm{bpm}(S D=0.94$; range $=-3.3$ to 2.6 ). The difference did not correlate with the mean heart rate $\left(\mathrm{Mean}_{\mathrm{SSG}, \mathrm{ECG}}\right)(r=-.16 ; p=$ $.17)$, but the absolute difference ( $\left(\mid\right.$ Diff $\left._{\mathrm{SSG}-\mathrm{ECG}} \mid\right)$ did $(r=$ $.42 ; p=.0002)$, suggesting an impact of the heart rate itself on the validity of measurements.

The greatest agreement between measurements was reached in the supine condition: The mean difference here was $0.1 \mathrm{bpm}(S D=0.75$; range $=-2.09$ to 1.12$)$, in contrast with the sideways condition (mean $=0.23 \mathrm{bpm}$; $S D=1.17$; range $=-3.3$ to 1.72 ) and the prone condition (mean $=0.65 \mathrm{bpm} ; S D=0.8$; range $=-0.93$ to 2.6), respectively. To elucidate the effects of the lying position and several subject variables such as gender, height, weight, and the subject's mean heart rate, two GLM analyses for repeated measures were computed, the first thereof on $\left|\operatorname{Diff}_{\mathrm{SSG}-\mathrm{ECG}}\right|$ and the second on Diff $_{\text {SSG-ECG }}$ as dependent variable. In both analyses, gender was the between-subjects factor, and height, weight, and the subject's heart rate were covariates. The heart rate was defined as the average of Mean ${ }_{\mathrm{SSG}, \mathrm{ECG}}$ across the three conditions. In both analyses, no significant effect of the lying position $[F(1,8)=0.3, p=.715$, and $F(1,8)=$ $0.84, p=.43]$ was found, but the results did show a significant effect of heart rate on $\mid$ Diff $_{\text {SSG-ECG }} \mid[F(1)=19.89$, $p=.0003]$. 
To assess the amount of variability in accuracy that is attributable to subjects and lying positions, two further ANOVAs, again on $\mid$ Diff $_{\text {SSG-ECG }} \mid$ and on Diff ${ }_{S S G-E C G}$, with the lying position as fixed and the subject as random factor, were performed. The only significant effect was found for the factor subject $[F(23,46)=1.9, p=.03]$, in the case of Diff ${ }_{\mathrm{SSG}-\mathrm{ECG}}$ as dependent variable.

Limits of agreement. The values for the $95 \%$ confidence boundaries of the limits of agreement in the population were calculated the same way as in Experiment 1 (Formula 1). The values for Experiment 2 were slightly worse: -2.23 to $-0.9 \mathrm{bpm}$ for the lower limit and 1.55 to $2.88 \mathrm{bpm}$ for the upper limit, respectively.

\section{VALIDATION EXPERIMENT 3}

To assess the accuracy of the respiration rate measurements, a third small validation experiment was carried out. Seven male and 7 female voluntary subjects (mean age $=$ $31.86, S D=8.1$; mean height $=172.6 \mathrm{~cm}, S D=11.5$; mean weight $=67 \mathrm{~kg}, S D=11.5$ ) lay on the test bed in either a supine, sideways, or prone position for $5 \mathrm{~min}$ each. The respiratory movements were recorded by measuring both the circumference over the chest and the circumference over the abdomen with two strain gauges (silastic tubes). Respiratory rates were computed by counting the cycles in the abdominal signal, because people in a relaxed state usually breathe using predominantly abdominal movements. Across all conditions, the mean rate by SSG was $12.45(S D=3.39$; range $=7.08-18.84)$ and by the strain gauge, it was $12.41(S D=3.55$; range $=5.8-18.6)$. The overall mean difference between both measurement methods was $0.03(S D=0.33)$. The mean difference was smallest in the sideways condition $(0.005, S D=0.25)$, but not significantly different from the other conditions. Among the 42 different cases (14 subjects, three experimental conditions), one outlier was detected with a difference larger than 1 . The $95 \%$ confidence intervals for the limits of agreement in the population (Formula 1) were calculated to be -0.95 to -0.31 and 0.37 to 1.01 , using all cases, and -0.78 to -0.21 and 0.17 to 0.78 , omitting the outlier.

The detection of cessations of respiratory activity during sleep - so-called sleep apneas - is of major concern to most sleep physicians. It would therefore be practical if such apneas could be detected with SSG. Apneas typically last between 30 and $60 \mathrm{sec}$, and periods of apnea, which are usually accompanied by a decrease in heart rate, can in principle be identified in the respiratory (and heart) pattern signals. We verified this possibility by asking the subjects in each case to intentionally stop breathing for about $30 \mathrm{sec}$ while their SSG signals were recorded normally.

\section{DISCUSSION}

\section{Heart Activity}

By increasing the number of subjects to 24 in Experiment 2 , we were able to show that different subjects and different heart rates account for most of the variability in accuracy. However small this variability might be, we suggest that, as with, for example, actigraphy, the measurement of the heart rate by SSG is probably most sensitive when a within-subjects design is used. From the results of the validation experiments, we can infer that potentially unwanted influences of different bed constructions on the validity of measurements are likely to be negligible and that the lying position has only a minor effect on measurement accuracy. In Experiment 2, a fairly consistent measurement bias of $+0.33 \mathrm{bpm}$, on average, was observed. This deviation can easily be corrected for in the future by adjusting the measurement algorithm. The confidence intervals for the limits of agreement were slightly larger in Experiment 2. This is most likely explained by the greater variance and somewhat greater range of differences in the prone and sideways conditions when compared with the supine-only measurements in Experiment 1 . The conditions in Experiment 2 with many subjects were similar to an intended application situation for SSG, and were therefore representative. We thus conclude that the confidence boundaries from Experiment 2 apply and that measurement by SSG would, in $95 \%$ of the observations, yield a heart rate that does not deviate more than $2.23 \mathrm{bpm}$ below and not more than $2.88 \mathrm{bpm}$ above the true value.

We are aware that our validation does not cover the full range of possible agreement parameters. For example, the length of the epoch over which the heart rate is calculated is inherently related to accuracy. Reducing the epoch length to half a minute or a few seconds, for example, will most probably yield less agreement between ECG and SSG. Also, the ability of SSG to extract individual beat-tobeat intervals with sufficient accuracy to define heart rate variability has not yet been investigated in detail.

\section{Respiration}

The comparison between respiration rates computed with SSG and an established method (strain gauge) in Experiment 3 shows that respiration rate measurement by SSG may also be feasible. However, if respiratory effort amplitudes are of interest, measurement with a strain gauge should be preferred. The level of accuracy of the respiration rate measurements is comparable to that of heart rate measurements. There is also no indication currently that SSG would fail to detect sleep apneas. However, no systematic experiments with clinical samples of apnea patients have been conducted to date.

The experiments described were conducted to provide a rough estimate of the accuracy one can expect when using SSG to measure heart and respiration rate. Whether SSG is accurate enough in a particular context must be decided on a case by case basis, depending on the type of research it might be used for.

\section{ACTIGRAPHY AND POSITION MEASUREMENTS}

\section{Actigraphy Measurements}

As already mentioned, the voltage outputs of the reflex light barriers in the sensors are split into AC and DC com- 
ponents to create the raw $\mathrm{AC}$ and the raw DC signal. Body movements of the subject appear in the AC component as well as in the DC component. Both sources can be used to infer actigraphy data. In the AC component, movements are characterized by sudden heavy deflections on all four channels, often overriding the dynamic range of the A/D conversion (which results in clipped signals). The DC component signals reflect the absolute force applied to the four bedposts and thus permanently monitor the center of gravity of the bed. Movements of the body or limbs give rise to a change in the center of gravity. Either the magnitude of that change can be computed-for example, every second - or a predefined threshold value of change can be employed to increment a counter. Whereas the DC component signals reflect larger body movements, the AC component is more sensitive and detects very small movements, for example, from a hand or foot. We currently employ three different measures to define actigraphy patterns: (1) The magnitude of change of the DC signals in each consecutive second, (2) a counter value based on a threshold for the change of the DC signals, and (3) the total number of clipped samples on all four channels in the raw AC signal within a certain time frame (e.g., $1 \mathrm{sec}$ ). Figure 6 pinpoints how actigraphy units are related to the $\mathrm{AC}$ and DC signals. The actigraphy units shown in panel D were calculated from the sum of the absolute differences between subsequent DC sample values of each channel in four consecutive sampling intervals, giving one actigraphy value per second.

During several experimental nights, actigraphy data obtained via SSG were compared with the data from a wrist actimeter (Model AW4 Actiwatch, Cambridge Neurotech
Ltd., U.K.). Since the methodology employed to calculate actigraphy units in SSG is, on the one hand, different from wrist actigraphy, and we, on the other hand, believe that SSG is more accurate during sleep, we would not expect congruency between the two methods, and we decided not to systematically examine their degree of agreement. One experimental night involving the parallel measurement of actigraphy with SSG and a wrist actimeter during 5001 -min epochs served as a showcase and provided the following results: Regardless of the magnitude of the actigraphy value, both methods agreed in $84 \%$ of the epochs on whether movement was present (actigraphy unit $>0$ ) or not. For 57 epochs, the actimeter assigned movements that were not detected by SSG, and in 23 epochs, SSG assigned movements that were not detected by the actimeter. This discrepancy is most probably caused by different threshold values for the movement detection but also because not necessarily the same movements are being recorded by an actimeter worn on one wrist and a whole bed that records vibrations regardless of where the movements originated.

There are a couple of known drawbacks in wrist actimeters that are not apparent in SSG-at least when the scope of actigraphy measurements is sleep behavior only. For example, the influence of the placement of the actimeter (waist vs. extremities) on the validity of the measurements has been subject to debate (van Hilten, Middelkoop, Kuiper, Kramer, \& Roos, 1993). Device placement is not an issue in SSG, because any kind of movement anywhere on the bed will be detected. SSG is also more accurate with regard to time than actimeters. The aggregation of movements over epochs of a given duration (e.g., $1 \mathrm{~min}$ )
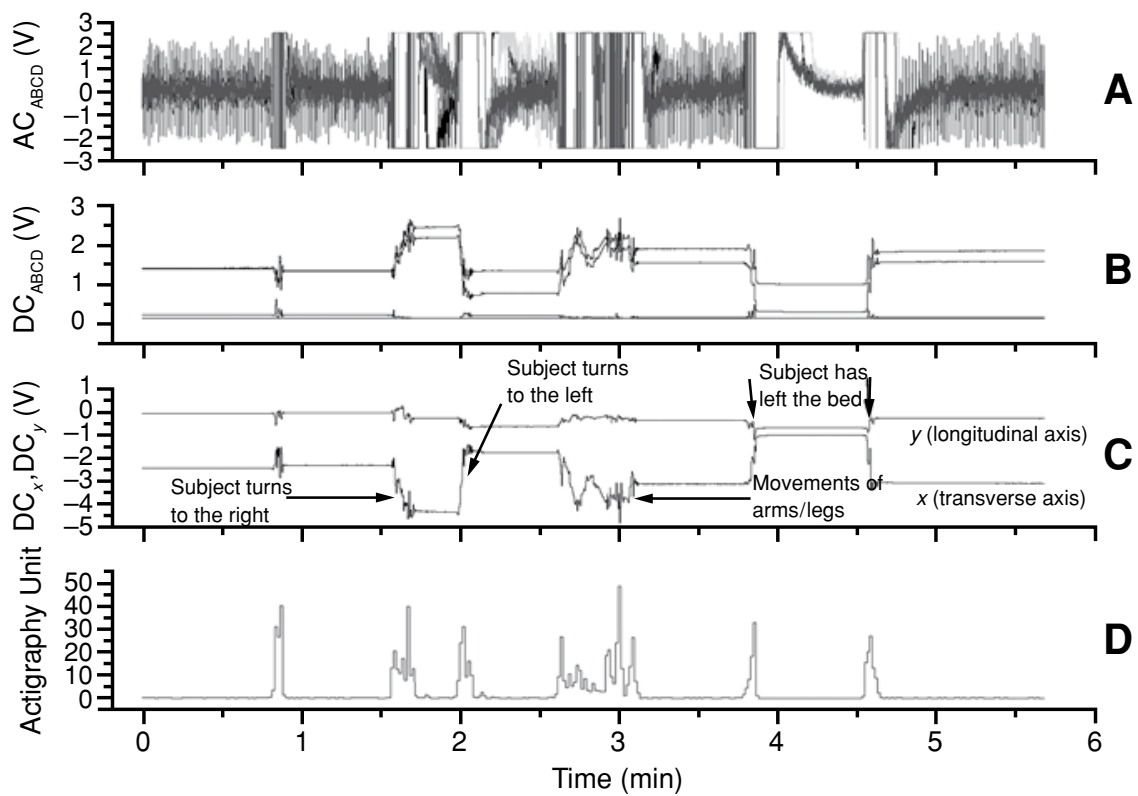

Figure 6. Actigraphy and position data in a 5.7-min controlled experimental session. (A) Raw AC signal, partially clipped due to movements of the subject. (B) Raw DC signal. (C) DC $x$ and $\mathrm{DC}_{y}$ signal. (D) Actigraphy units for consecutive seconds. 
usually employed in wrist actimeters does not allow the assignment of a particular single movement event to a precise point in time. Because SSG raw signals are sampled and stored at $100 \mathrm{~Hz}(\mathrm{AC})$ and $4 \mathrm{~Hz}$ (DC), respectively, every single movement is recorded immediately. This is especially useful for event-correlated data analysis, for example, in studies or experiments of noise-induced sleep disturbance. SSG can also easily ascertain bedtime and rise time, as well as interruptions of time in bed, by monitoring whether the total force on the sensors, as reflected in the DC component, increases or decreases rapidly. However, to make accurate between-subjects comparisons of actigraphy, extensive calibration procedures would be necessary for each particular installation. Therefore, actigraphy data gathered with SSG can, in most instances, only be analyzed reliably within the scope of within-subjects research.

\section{Position Measurements}

The DC signals not only reflect the magnitude of movements of the sleeper, but provide information about body turns and shifts of the sleeping position. The majority of these shifts appear in the transverse axis (in the $\mathrm{DC}_{x}$ signal), as a consequence of subjects shifting from one side of the bed to the other (Figure 6, panel C). In one experimental study about sensitivity to electromagnetic fields and sleep behavior, which employed our first prototypes, the DC signals could even be used to elegantly measure (unconscious) evasion by the subjects of the source of the field (Müller, 2000).

\section{CONCLUSIONS}

SSG provides an easy and unobtrusive way of monitoring and recording basic life functions during sleep. The system we have developed is portable and cost effective, and was constructed from easily available components. The main advantages of SSG are that no parts (cables, electrodes, or mats) are in direct contact with the body of the subjects and that the method does not impose any restrictions on freedom of movement. The proper installation of the system and placement of the sensors takes about $30 \mathrm{~min}$, but after the sensors are firmly installed under a particular bed, they can remain there permanently. No attending staff is needed further, which is a significant economic factor. The system thus primarily caters to the field researcher who needs a robust tool to assess sleepquality patterns in long-term studies without requiring subjects to handle any part of the measuring equipment themselves. Other domains of application might include the monitoring of sleep behavior of elderly people or of patients or subjects who cannot (or do not want to) wear electrodes or wrist actimeters, for whatever reason.

A few shortcomings of the system in its current state have not yet been overcome. Our prototypes have only been tested with normal single beds, of the type broadly used in Europe. But construction of beds and type of mattresses might differ considerably across countries or cultures. Thus, the applicability of the method might be limited. There is also, as a matter of principle, no way to trace the heartbeat or the respiration movement when a subject's motor activity level on the bed is above a certain threshold, a disadvantage that SSG has in common with all other "sensitive bed" methods. Furthermore, we still observe considerable lack of agreement between the interbeat-interval variability measures of SSG and ECG. One reason for this is that SSG and ECG do not measure the exact same thing, but different aspects (displacement of body mass vs. electric potential difference changes) of the same physiological event. More detailed examinations of the temporal relation between ECG curves and SSG signals, similar to the ones carried out by Goedhard (1979) with BCG signals, will be needed for a full understanding of the mechanical and biomechanical laws that underpin SSG recordings.

Although SSG can be used as a full-fledged alternative to established methods of actigraphy and respiration measurement, the practical limitations of its usability pertain to the maximum detectable heart rate (currently, $120 \mathrm{bpm}$ ) and the accuracy of heart rate measurements $( \pm 2-3 \mathrm{bpm}$ tolerance). Our system therefore might not meet the requirements for the investigation of clinical samples, for example, comprising patients with tachycardia or tachyarrhythmia. But in most other cases, the level of precision and the detectable range of heart rate between 31 and $120 \mathrm{bpm}$ is sufficient to derive sleep-stage-related heart rate dynamics or event-correlated changes in activation during sleep. But we must make it clear that SSG is not a replacement for PSG as it is used in sleep laboratories. In a clinical context, SSG may rather be employed as a screening tool in the natural environment of patients with sleep disorders, before PSG comes into place. However, our long-term objective is to provide the hardware and software for an automated sleep-stage analysis (or an approximation thereof) based entirely on contact-free measurements. As a first step, the circuit on the sensors will be improved in order to reduce unwanted noise generated by the electronics. Second, a new adaptive signal analysis algorithm, based on factor graphs - to enhance the detection of single heartbeats, also from weak or confounded signals - is currently being developed.

\section{REFERENCES}

Alihanka, J., \& Vaahtoranta, K. (1979). A static charge sensitive bed. A new method for recording body movements during sleep. Electroencephalography \& Clinical Neurophysiology, 46, 731-734.

Alihanka, J., Vaahtoranta, K., \& SaArikivi, I. (1981). A new method for long-term monitoring of the ballistocardiogram, heart rate, and respiration. American Journal of Physiology, 240, R384-R392.

Bland, J. M., \& Altman, D. G. (1986). Statistical methods for assessing agreement between two methods of clinical measurement. Lancet, 1, 307-310.

Crisp, A. H., Stonehill, E., \& Eversden, I. D. (1970). The design of a motility bed including its calibration for the subject's weight. Medical \& Biological Engineering, 8, 455-463.

Goedhard, W. J. (1979). Ballistocardiography: Past, present and future. Bibliotheca Cardiologica, 37, 27-45.

GoRDON, J. W. (1877). On certain molar movements of the human body produced by the circulation of the blood. Journal of Anatomy \& Physiology, 11, 533-536. 
HENDERSON, Y. (1905). The mass-movements of the circulation as shown by a recoil curve. American Journal of Physiology, 14, 287-298.

MüLLER, C. H. (2000). Projekt NEMESIS: Niederfrequente elektrische und magnetische Felder und Elektrosensibilität in der Schweiz. Unpublished doctoral dissertation, Swiss Federal Institute of Technology, Zürich, Switzerland, No. 13903, from e-collection.ethbib.ethz $. \mathrm{ch} /$ show?type $=$ diss\&nr $=13903$

Muzet, A., Becht, J., Jacquot, P., \& Koenig, P. (1972). A technique for recording human body posture during sleep. Psychophysiology, 9, 660-662.

Proakis, J. G., \& Manolakis, D. G. (1996). Digital signal processing: Principles, algorithms, and applications (3rd ed.). Upper Saddle River, NJ: Prentice Hall.

Schierz, C., Müller, C. H., Krueger, H., \& Honegger, H. (2003). Swiss Patent No. 693,300. Zurich, Switzerland: Eidgenössische Technische Hochschule Zürich.

SIIVOLA, J. (1989). New noninvasive piezoelectric transducer for record- ing of respiration, heart rate and body movements. Medical \& Biological Engineering \& Computing, 27, 423-425.

Sмiтн, S. W. (1997). The scientist and engineer's guide to digital signal processing. San Diego: California Technical Publishing.

Starr, I., Rawson, A. J., Schroeder, H. A., \& Joseph, N. R. (1939). Studies on the estimation of cardiac output in man, and of abnormalities in cardiac function, from the heart's recoil and blood's impacts; the ballistocardiogram. American Journal of Physiology, 127, 1-28.

van Hilten, J. J., Middelkoop, H. A., Kuiper, S. I., Kramer, C. G., \& Roos, R. A. (1993). Where to record motor activity: An evaluation of commonly used sites of placement for activity monitors. Electroencephalography \& Clinical Neurophysiology: Electromyography \& Motor Control, 89, 359-362.

(Manuscript received December 10, 2004; revision accepted for publication April 5, 2005.) 